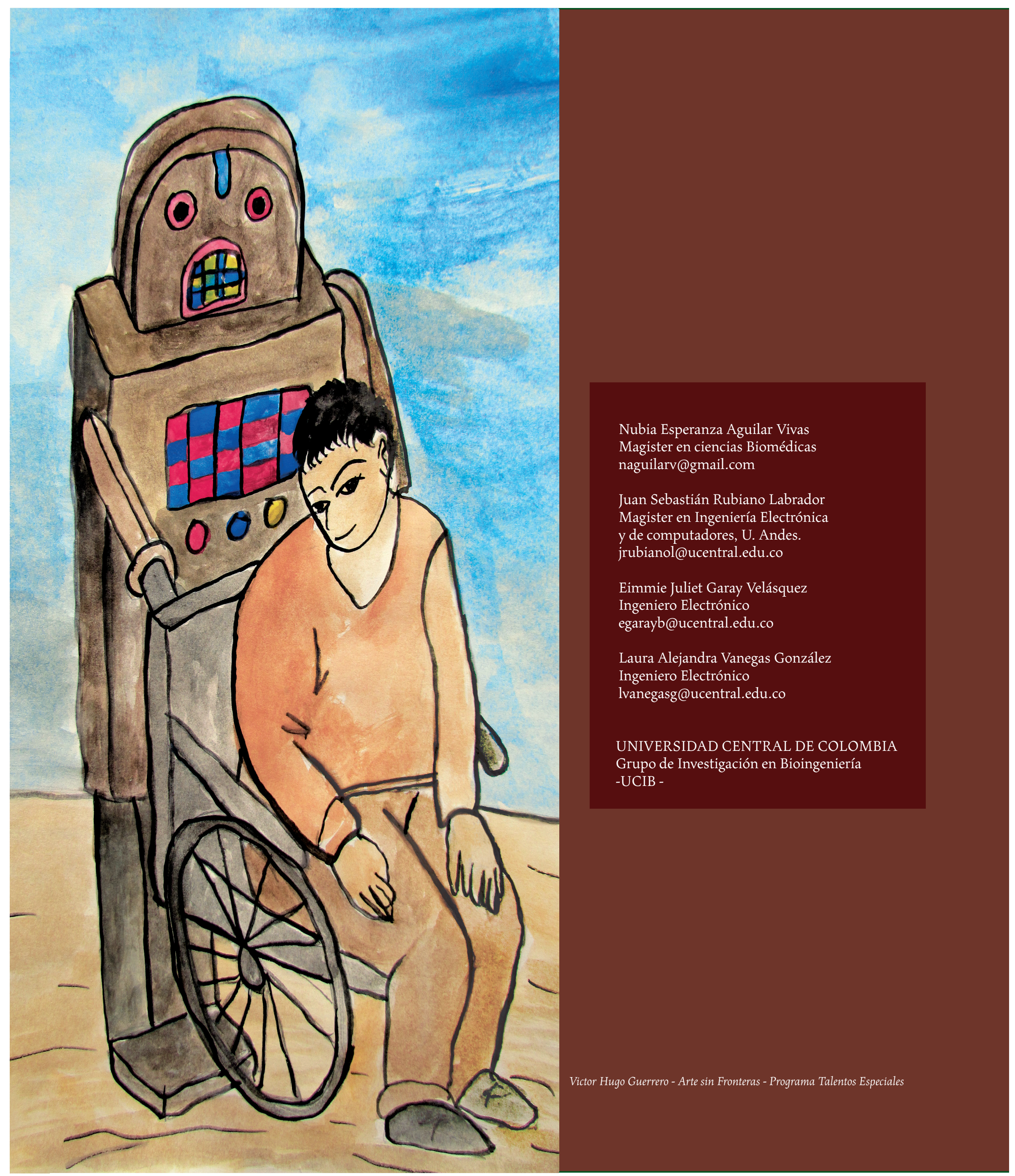




\section{DOMÓTICA ASISTENCIAL: UN DESARROLLO TECNOLÓGICO DE APOYO A LA DISCAPACIDAD}

\section{Home Automation Care: a Technological Development of Disability Support}

Fecha de Recepción: 17 de Febrero de 2011 - Fecha de Aprobación: 16 de Agosto de 2011

\section{RESUMEN}

El presente artículo muestra el desarrollo de un sistema tecnológico en el área de la Domótica asistencial, esta área se ocupa de desarrollar ayudas para automatizar la vivienda de personas en condición de discapacidad, con el propósito de mejorar su calidad de vida. El sistema desarrollado utiliza como señal de entrada, las señales electromiográficas (EMG) de dos grupos musculares (flexor radial del carpo y masetero), por medio de estas señales, se controla un sistema electrónico capaz de dar órdenes para Encender/apagar la luz y Encender/apagar una alarma. El sistema utiliza la señal digitalizada como comando y controla las variables citadas, utilizando una red neuronal que ejecuta la labor asignada. Este dispositivo se convierte en una propuesta de desarrollo tecnológico de bajo costo, útil como apoyo a la discapacidad y al mejoramiento de la calidad de vida.

PALABRAS CLAVES

Domótica asistencial, discapacidad, electromiografía, Red Neuronal Artificial (RNA), rehabilitación.

\section{ABSTRACT}

This paper shows the development of a technological system in the area of Home Automation care, this area is concerned with developing automated support for the housing of people living on disability, in order to improve their quality of life. The implemented system uses as input signal, the electromyographic signals (EMG) of two groups of muscles (flexor carpi radialis and masseter), through these signs, you control an electronic system able to give orders for on / off light Turning on the alarm. The system uses the digitized signal as a command and controls these variables, using a neural network that performs the work assigned. This device becomes a proposed low-cost technology development, useful as an aid to disability and improving quality of life.

KEY WORDS

Home Automation care, disability, electromyography, Artificial Neural Network, Rehabilitation. 

no se trata de un hecho específico, sino de una serie de procesos asociados a la evolución de sistemas electrónicos y de computación, que permiten el desarrollo de tecnologías aplicadas al "Domus", es decir al hogar o vivienda. En la actualidad este concepto es a lo que se le denomina la "casa del futuro", o el "Hogar Digital", se refiere a una propuesta innovadora de tecnología asociada al entorno que puede facilitar al discapacitado una mejor condición de vida, además soluciona algunas de las necesidades más apremiantes del adulto mayor, o de cualquier persona dependiente directamente en su propio domicilio, también ofrece bienestar, facilitando en el discapacitado un mayor control voluntario de su entorno gracias a la automatización de su hogar y/o entorno de trabajo. Se presume que se inicia a finales de los años 70 y desde entonces se han desarrollado diversos dispositivos que suplen necesidades a un usuario en diferentes entornos. Una de las principales aplicaciones de la Domótica es en el área de la asistencia a usuarios en condición de discapacidad $(1,2,3,9,5)$, estas aplicaciones permiten entre otros propósitos, hacer un uso eficiente de la energía, aportar seguridad, confort, y comunicación al usuario del sistema.

Estos sistemas tecnológicos toman una señal de entrada captada por sensores específicos, procesan dicha señal y como salida se activan o desactivan los actuadores, que ejecutan la respuesta deseada.

El uso de señales bioeléctricas superficiales provenientes de los músculos (sEMG) ha aumentado en los últimos 10 años, por medio de esta técnica se registran diferencias de potencial eléctrico que tienen muy baja intensidad, desde $50 \mu \mathrm{V}$ a $5 \mathrm{mV}$, esto muestra que se requieren sistemas electrónicos de amplificación para convertirlos en señales utilizables $(6,7)$. Dichas señales pueden ser generadas por diversos movimientos como por ejemplo flexo-extensión, entre otras. A su vez se utilizan de formas diferentes, y su estudio y análisis puede ser un indicador como medida del esfuerzo muscular, y/o para evaluar el músculo en respuesta a estímulos externos. De acuerdo a su uso se debe diseñar para obtener este tipo de señales un sistema de adquisición que incluye las etapas de pre-filtrado y acondicionamiento de la señal $(8,9,10,11,12)$.

En este artículo se presenta un sistema electrónico que adquiere y emplea señales EMG superficiales de dos grupos musculares, para procesarlas y generar una codificación que es utilizada como comandos para controlar en una vivienda algunos elementos que hacen parte de la vida cotididana del usuario, garantizando confort y seguridad para este. A su vez este trabajo ayuda a mejorar su calidad de vida y promueve la inclusión social ofreciéndoles la posibilidad de sentirse independientes en su vida diaria. El desarrollo de dispositivos de control domótico son la tecnología actualmente utilizada para facilitar a las personas su desempeño domiciliario y/o laboral, en la domótica se encuentran dos vertientes: viviendas inteligentes y la domótica aplicada a la accesibilidad. La domótica aplicada a la accesibilidad para discapacitados es una rama que se dedica a "generar dispositivos que faciliten la integración de una persona con algún tipo de discapacidad con el entorno en que se desenvuelve". Este trabajo corresponde a un desarrollo aplicado a la domótica de accesibilidad donde se usan señales electromiográficas como comando para estos dispositivos $(13,14)$.

El desarrollo de este sistema implementa técnicas de procesamiento de señales y caracterización basadas en Wavelets, FFT y aproximación temporal donde posteriormente se reconocerán los patrones de movimiento de los dos grupos musculares, masetero y flexor radial del carpo, mediante Redes Neuronales Artificiales. El dispositivo biomédico realizado es ajustable a cada paciente mediante una fase de entrenamiento regida por un protocolo de movimientos debido a las diferentes características de las señales electromiográficas de un mismo músculo y movimiento en distintos individuos.

\section{METODOLOGÍA}

El desarrollo de este proyecto comprende diferentes fases las cuales se mencionan a continuación:

Fase 1 - Elección de grupos musculares: La escogencia de los músculos, se hizo luego de estudiar cinco grupos musculares, ubicados en diversas partes del cuerpo, en los cuales se estudio la señal EMG en personas sin discapacidad, donde los criterios utilizados en la selección fueron: las mejores características de amplitud (voltaje) y forma de la señal. Para el estudio y obtención de las señales EMG se utiliza inicialmente un sistema comercial de adquisición de señales biomédicas (Biopac Lessons y Biopac 3.7) (15). La elección de estos músculos obedece también a que se necesitan probar diversas localizaciones del músculo generador de señales, pues el dispositivo está dispuesto para ser implementado en personas con discapacidad motora, tipo hemiplejia o cuadriplejía, que tengan por lo menos un músculo en cualquier localización, que sirva como generador de señales EMG ya que este sistema electrónico puede ser utilizado en cualquier músculo que este activo, es decir que pueda generar una señal bioeléctrica, con el fin de que personas en condición de discapacidad motora severa también puedan usarlo.

Fase 2 - Canales EMG: A partir del estudio y elección de los grupos musculares trabajados en la fase anterior, se diseña la etapa de adquisición con dos canales EMG de entrada. Para cada una de las pruebas se ubican los electrodos en los músculos correspondientes (Ver figura 1), dos electrodos a una distancia aproximada de 3 centímetros entre ellos y en la disposición de las fibras musculares y un tercer electrodo que funciona como electrodo de referencia, colocado en otro sector que no sea el del músculo examinado $(16,17)$.

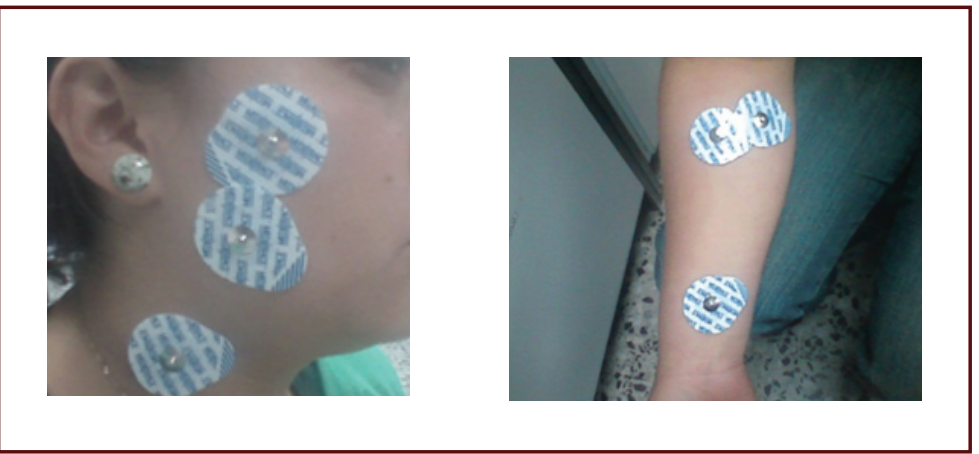

Figura 1. Ubicación de los electrodos en los músculos escogidos, Masetero y flexor radial del Carpo. 
Cada canal EMG está constituido por los siguientes etapas: etapa de Pre-amplificación de la señal en la superficie de la piel, con lo cual se logra la obtención de una señal EMG confiable. Pre-amplificador de instrumentación diferencial, que atenúa las señales indeseables en el entorno, etapa de Aislamiento y protección interna de descarga electrostática, esta etapa está constituida por un circuito que aísla al paciente de cualquier flujo de corriente externa y la etapa de Filtrado de las señales electromiográficas, pues las señales que provienen de los músculos tienen un rango de frecuencias en el cual se concentra la mayor parte de su energía. El filtrado de la señal garantiza que se conservarán los componentes de la señal que cuenten con la mayor energía. En general, la mayor parte de la energía se concentra entre los $30 \mathrm{~Hz}$ y los $500 \mathrm{~Hz}(18,19,20,21,22)$.

Fase 3 - Digitalización señales EMG: Se crea una base de datos con las señales EMG digitalizadas por medio de un microcontrolador con una frecuencia de muestreo de $1000 \mathrm{~Hz}$ y un ADC de 10 bits, el cual se conecta con el computador por la interfaz RS-232 (23).

Fase 4 - Extracción de características de las señales EMG e implementación de la RNA: Una vez digitalizadas las señales EMG, se hizo necesaria la implementación de la transformada Wavelet; la implementación de esta transformada permite la extracción de un número de coeficientes que indican las características temporales y espectrales de la señal EMG (24). La transformada Wavelet implementada en el software MATLAB permitió asignar a cada movimiento realizado un rango de valores de energía, RMS (Root Mean Square).

Adicionalmente se diseña e implementa una Red Neuronal Artificial (RNA) que recibe cada uno de los valores de cada señal e inicia una fase de entrenamiento, en el cual las neuronas que forman parte de la red "aprenden" que a cada rango de valores de entrada (valores correspondientes a la señal), les corresponden una salida específica, en el caso tratado en este trabajo seria el proceso de encendido y apagado de dos dispositivos del hogar (luz del cuarto y una alarma).

Esta red fue usada de manera repetitiva para asegurar su nivel de funcionamiento y calcular el porcentaje de asertividad. Luego el usuario realiza los movimientos indicados y la red se encarga de reconocer los patrones de movimiento, y por consiguiente se genera una codificación personalizada que permite controlar algunos elementos en el interior de una vivienda, que aseguran confort y seguridad para el usuario.

El prototipo desarrollado en el presente trabajo es un primer modelo funcional donde se busca realizar las pruebas en personas que no tienen discapacidad para así generar un protocolo de movimientos asociados a las señales EMG de cada grupo muscular y a su vez los cambios necesarios para realizar un ajuste personalizado del dispositivo. En un siguiente estudio se pretende tener la participación de personas con diferentes tipos y grados de discapacidad, como también de personal especializado (médicos y fisioterapeutas) para realizar un estudio ampliado del funcionamiento del prototipo implementado y medir la eficiencia de este en diferentes situaciones del entorno.

\section{RESULTADOS}

El cumplimiento de la Fase 1 permitió analizar con el software Biopac Lessons y Biopac 3.7 las diferentes señales de 5 posibles músculos generadores, este trabajo preliminar fue útil en la toma de decisiones de los grupos musculares con mejores características y también en la definición de la frecuencia de muestreo $(1000 \mathrm{~Hz})$ utilizada en el diseño del dispositivo.

En la Fase 2 a la 4, a partir de los resultados de las pruebas preliminares se diseñó y construyó un dispositivo electrónico con la capacidad de adquirir, procesar y codificar las señales electromiográficas (EMG) provenientes de dos grupos musculares elegidos: flexor radial del carpo y masetero (Ver figura 4).

Las señales obtenidas con el dispositivo diseñado permitieron corroborar la similitud de estas con las obtenidas con el sistema Biopac, lo que se constituye en una buena referencia para comparar el funcionamiento del dispositivo diseñado, teniendo en cuenta que este es un sistema de obtención de señales biomédicas avalado a nivel internacional (25).

Los principales movimientos realizados con el músculo masetero de apertura y cierre de boca, se utilizaron como comandos para el control de apagado y encendido de luz, y en el caso del músculo flexor radial del carpo se realizaron movimientos de la mano y el dedo pulgar para el control del encendido y apagado de una alarma.

Las siguientes son las imágenes de las señales electromiográficas adquiridas de los dos músculos seleccionados, Flexor radial del carpo y masetero (Ver figura 2 y 3 respectivamente), lo que permitió el reconocimiento de las características de la señal de cada musculo estudiado.

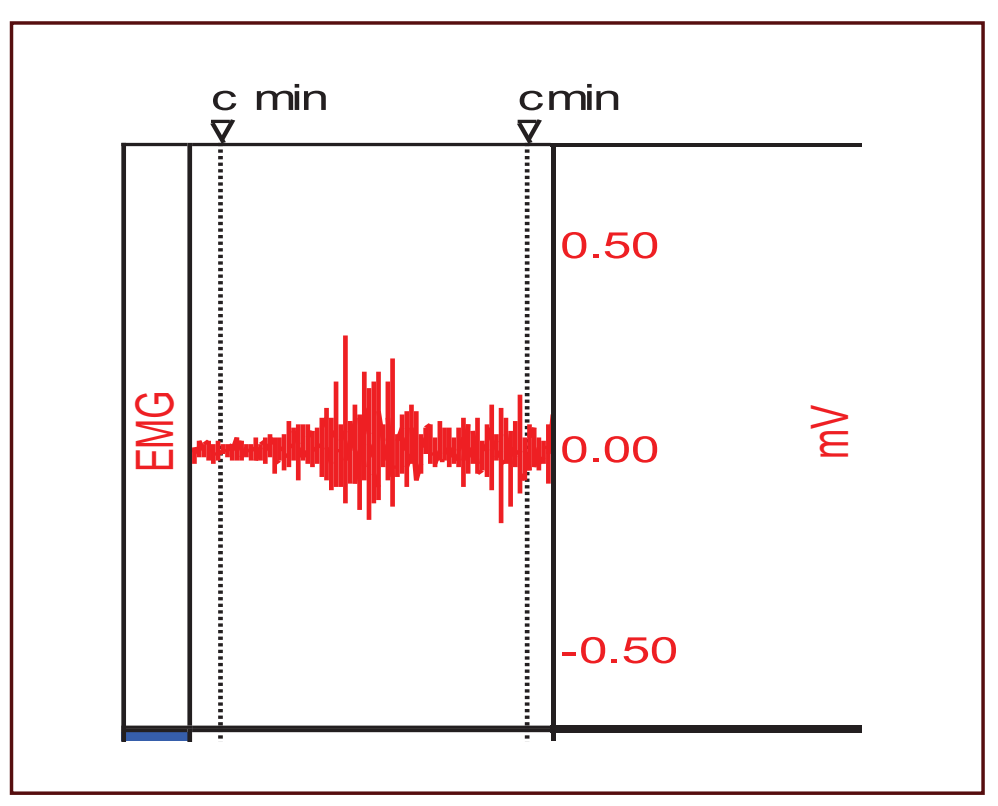

Figura 2. Señal EMG del músculo flexor radial del carpo. 


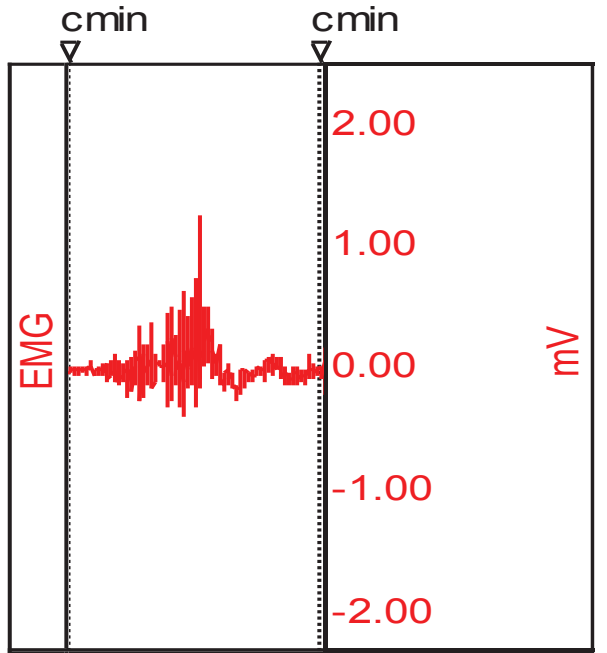

Figura 3. Señal EMG del músculo masetero.

Una vez la señal electromiográfica es digitalizada, se encuentra que las señales electromiográficas tienen una gran similitud, y por lo tanto son difícilmente discriminables. Debido a esto fue fundamental el extraer las características temporales y espectrales de las señales EMG mediante la transformada Wavelet y dar una entrada diferenciable a la RNA (Ver figura 4).

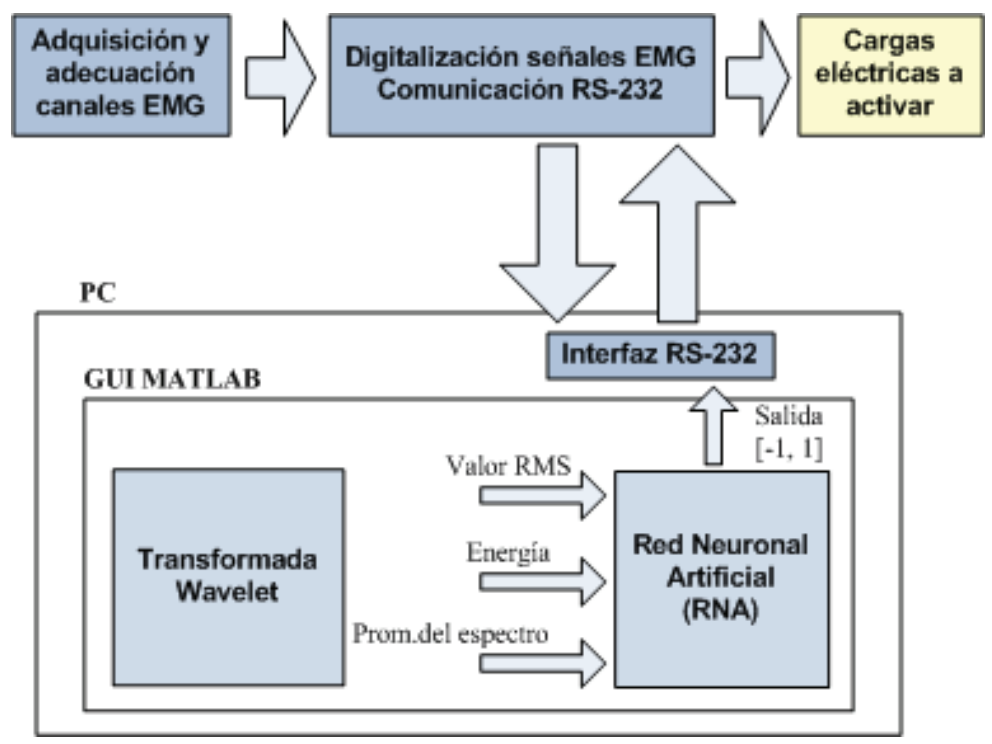

Figura 4. Diagrama de bloques del sistema de control domótico controlado por señales $E M G$.

Luego del desarrollo del prototipo en mención, este se pone a prueba, puesto que es necesario conocer el porcentaje de aciertos en la ejecución de un movimiento específico, para determinar la eficiencia del dispositivo, se realizan pruebas en el Laboratorio de Electrónica de la Universidad Central de Bogotá, los resultados se muestran en la Tabla 1, allí se aprecia el número de aciertos durante 14 pruebas realizadas en un sujeto sano, de 21 años de edad, sin discapacidad. Los valores 1 y o se utilizan para indicar si el dispositivo acertó (26) o no (o) en la identificación del movimiento realizado. Se observan
14 intentos hechos por el sujeto 1 utilizando el dispositivo. En la columna "Movimiento realizado" se muestra el movimiento ejecutado por el usuario del dispositivo. En la columna "Movimiento identificado", se muestra el movimiento que el dispositivo reconoce.

\begin{tabular}{|c|c|c|c|c|c|c|c|c|c|c|c|c|c|c|c|}
\hline $\begin{array}{l}\text { MOVIMIENTO } \\
\text { REALIZADO }\end{array}$ & & MO & VI & 9 & IE] & NT & & & & & FI & CA & DO & & $\begin{array}{c}\% \\
\text { ACIERTOS }\end{array}$ \\
\hline Flexión de la muñeca & 1 & 1 & 1 & 1 & 0 & 1 & 1 & 1 & 1 & 1 & 1 & 1 & \begin{tabular}{|l|l}
1 & \\
\end{tabular} & 1 & 92,86 \\
\hline $\begin{array}{l}\text { Flexión del dedo pulgar } \\
\text { hasta la base del meñique }\end{array}$ & 1 & 1 & 1 & 1 & 0 & 1 & 1 & 1 & 0 & 1 & 1 & 1 & 1 & 1 & 85,71 \\
\hline Ningún movimiento & 1 & 1 & 1 & 1 & 1 & 1 & 1 & 1 & 1 & 1 & 1 & 0 & 1 & 1 & 92,86 \\
\hline Movimiento de sonreír & 1 & 1 & 1 & 1 & 1 & 1 & 1 & 1 & 1 & 0 & 1 & 1 & 1 & 1 & 92,86 \\
\hline $\begin{array}{l}\text { Cierre de boca con fuerza } \\
\text { de mordida aplicada en } \\
\text { molares }\end{array}$ & 0 & 0 & 1 & 1 & 0 & 1 & 1 & 1 & 0 & 1 & 1 & 1 & 1 & 1 & 71,43 \\
\hline Apertura máxima de boca & 1 & 1 & 1 & 1 & 1 & 1 & 1 & 1 & 1 & 1 & 1 & 1 & \begin{tabular}{l|l}
1 & \\
\end{tabular} & 0 & 92,86 \\
\hline
\end{tabular}

Tabla 1. Porcentaje de aciertos en el dispositivo.

\section{DISCUSIÓN}

En Bogotá, según el Censo de población y vivienda de 2007 se identificaron 331.301 personas con limitaciones permanentes, de las cuales 28.679 viven en la localidad de Ciudad Bolívar, 23.840 viven en Bosa y 22.345 viven en San Cristóbal, siendo estas tres las localidades en donde más número de personas cuentan con algún tipo de limitación física (27). Del total de personas discapacitadas en Bogotá, el 32,7\% requiere de la ayuda permanente de una persona para su desenvolvimiento cotidiano, es decir, bañarse, vestirse y alimentarse a sí mismos, puesto que la principal función corporal afectada es la del movimiento del cuerpo, manos, brazos y piernas. A este hecho debe añadirse que las localidades anteriormente mencionadas, hacen parte de los estratos uno, dos y tres, y que esto hace que sea difícil acceder a un dispositivo médico que les permita por si mismos tener un control para manipular equipos, electrodomésticos y otros elementos asociados a su entorno.

Estas cifras bastante preocupantes nos llevan a pensar en un sistema que permita la autonomía mediante el control voluntario de elementos del entorno utilizando señales bioeléctricas provenientes del músculo esquelético, es claro que para este propósito se hace necesario el desarrollo de un dispositivo electrónico completo que permita no solo la obtención de la señal EMG en cada usuario y en diferentes músculos de su cuerpo, sino también que la procese y genere una salida de control sobre algún elemento eléctrico del hogar.

En efecto dicho dispositivo fue diseñado, implementado, y verificado su funcionamiento general, pero una de sus principales características es que este puede ser personalizado y fácilmente adaptado a cada usuario (con o sin discapacidad), sin embargo aunque el propósito es ayudar a mejorar la condición de discapacidad del usuario final este primer prototipo funcional fue probado exclusivamente en sujetos sanos.

Antes de que el sistema electrónico vaya a ser utilizado por un usuario, es necesario desarrollar en él un estudio del músculo o músculos más adecuados para activar las salidas de control, es decir utilizar un músculo funcional en donde sea fácil la ubicación de los electrodos, que corresponda a la estructura con mayor movilidad en cada caso y de acuerdo a cada tipo de discapacidad, con el fin de caracterizar los movimientos y ajustar el dispositivo de manera óptima 
y personalizada. Es por esta razón que se implemento una Red Neuronal Artificial donde esta puede someterse a una fase de entrenamiento ante un nuevo usuario, quien debe desarrollar un protocolo de movimientos con el fin de hacer un registro de las señales EMG provenientes de sus grupos musculares.

El uso del software MATLAB clasifica los valores y determina qué movimiento fue realizado. En este programa también se desarrolla un algoritmo para el entrenamiento y la ejecución de la red neuronal. La utilización de este software ha sido recurrente en trabajos como el de Betancourt y Giraldo (28), quienes lo emplean para aplicar la transformada Wavelet a los datos obtenidos del músculo bíceps y también para entrenar y ejecutar una red neuronal que permite el control de un brazo robótico, y por Aguilar (29) para implementar una red neuronal de propagación inversa y analizar la distinción de movimientos en pacientes con miembros amputados.

Las pruebas realizadas para la comprobación del funcionamiento adecuado y del número de aciertos entre los movimientos realizados y los identificados por el dispositivo se muestran en la Tabla 1, con esta tabla también se muestra que el dispositivo cumple con la activación y desactivación de los elementos a controlar en el diseño ya que se observa como tiene una efectividad máxima del 92,86\% y mínima de $71,43 \%$. Las pruebas realizadas son preliminares donde se comprueba que el prototipo desarrollado es completamente funcional, aunque se aplicó en personas sanas se evidencia que al ser personalizado puede ser adaptable a personas en condición de discapacidad física lo cual será motivo de un estudio posterior.

En el dispositivo motivo de este estudio se comprueba la utilidad de las señales EMG como comando para construir una interfaz entre el usuario y los elementos del entorno a controlar, se conocen algunos dispositivos que han sido desarrollados como interface hombre-máquina en los que se dan comandos por movimientos específicos de cualquier musculo, faciales, de mano, antebrazo, etc. que permiten controlar objetos como robots, silla de ruedas, prótesis denominadas mioeléctricas (30), sistemas Miofeedback (31), dispositivos de entretenimiento, electrodomésticos de uso diario, sistemas de evaluación de expresión emocional a nivel facial (32), entre otros. En el 2007 se desarrollo con estudiantes de Ingeniería electrónica de la Universidad Central un sistema que por medio de señales EMG controla un generador de voz para discapacitados del habla, y desde entonces se han desarrollado diversos trabajos utilizando estas señales $(33,34,35,36,37,38)$.

Actualmente las señales de entrada utilizadas con propósitos asistenciales son diversas, no solo señales EMG, sino que pueden usarse señales visuales, como en el caso de teléfonos con sensores y vibración para personas con discapacidad auditiva, y que también, poseen teclas grandes y sonido para personas en condición de discapacidad visual (39); otra señal de entrada puede ser el movimiento de la cabeza en donde se tiene interfaces inalámbricas que permiten controlar diferentes aparatos (FATRONIK) (40); la entrada de señal del iris puede controlar un ordenador (IRISCOM) (41). La entrada de voz puede usarse en un sistema de mando por infrarrojos, que controla todos los aparatos del hogar (SICARE LIGHT) (40); otros desarrollos utilizan como entrada las señales electrooculográficas para controlar el puntero del mouse, botones, teclado virtual y componentes de software (36), así mismo la entrada de señales electroencefalográficas pueden controlar una computadora de esta misma forma (25), en el diseño desarrollado en el presente trabajo se propone como señal de entrada, la señal EMG como una alternativa de control del dispositivo domótico (18).

En el mundo se conocen diferentes opciones de control de entorno, utilizando algunas de estas entradas de información citadas, en donde las acciones más frecuentemente controladas son: abrir/ cerrar la puerta, encender/apagar la luz, cambiar la posición de la cama, subir/bajar las persianas, controlar la temperatura, encender/ apagar la lámpara de mesa, entre otras (7). Estas acciones buscan mejorar la autonomía, fomentar la vida independiente, y dar al discapacitado una mayor seguridad ante imprevistos (2).

La aplicación de la Domótica es una excelente alternativa para mejorar la calidad de vida de las personas que habitan la vivienda tecnificada. Si bien, es cierto que los habitantes discapacitados tienen una amplia gama de deficiencias motoras, también es cierto que cualquiera de ellos tendría actividad al menos en un músculo generador de señales EMG. Gracias a la Domótica, personas que habitan en soledad y/o tienen problemas motores, pueden lograr una mayor comunicación con la familia o los cuidadores. Con estos desarrollos se obtiene una alternativa de monitoreo domiciliario, y estas posibilidades tecnológicas permiten que el individuo en condición de discapacidad pueda seguir viviendo en su casa, pero con una mayor libertad e independencia y un alto nivel de seguridad (2).

\section{CONCLUSIONES}

- El sistema diseñado reconoce las señales electromiográficas de manera personalizada y controla elementos del entorno del usuario en un vivienda, a su vez puede ser aplicado como una ayuda para individuos en condición de discapacidad.

- El sistema diseñado es capaz de controlar por medio de señales EMG el encendido/apagado de luz y el encendido/apagado de una alarma.

- La red neuronal implementada en este sistema hace que este pueda ser adaptado a cada usuario luego de una fase de entrenamiento que incluye un protocolo de movimientos específicos.

- Un sistema domótico es una ayuda tecnológica importante para mejorar la autonomía y la calidad de vida de un individuo en condición de discapacidad.

\section{Agradecimientos}

A la Universidad Central por permitir el uso de equipo de Laboratorio de obtención de señales biomédicas, a los estudiantes de pasantía en el Semillero de investigación "Desarrollo tecnológico en electrofisiología aplicada" del grupo de Investigación en Bioingeniería - UCIB por sus aportes en el mejoramiento del prototipo. 


\section{Referencia:}

\section{Las referencias a otras obras son una parte muy importante en la literatura científica; ya que estas permiten conocer más sobre los autores y}

mantener vivas sus voces dentro del texto.

1. REgATOS, R. Domótica accesible, Dossier 2006. [Documento electrónico]. URL: http://www.anobium.es/docs/gc_fichas/ doc/05FGOPVbgk.pdf [Consulta: 2010-08-12] . p. 15-17.

2. II Congreso Internacional sobre Domótica, Robótica y Teleasistencia para Todos Fundación ONCE para la Cooperación e Integración Social de las Personas con Discapacidad. 2008. Fundación ONCE para la Cooperación e Integración Social de las Personas con Discapacidad. 2008. [Documento electrónico]. URL: http://www.imsersomayores. csic.es/documentos/documentos/once-escaparate-01.pdf [Consulta: 2010-08-12]

3. MATHIASSEN, S. E. y Cols. Amplitude aspects and functional considerations on surface EMG electrode displacement with particular emphasis on the upper trapezius muscle". SENIAM. 1997. pp. 84. [Consulta: 2010-02-20].

4. RAMÍREZ A. y Cols. Análisis de sensibilidad por la colocación de los electrodos en la electromiografía de superficie (sEMG). Revista. Fac. Ing. Univ. Antioquia $\mathrm{N}^{\circ} 46$. Diciembre, 2008. p. 70-79.

5. COCERO O.E y Cols. Introducción a la electromiografía y a la conducción eléctrica del nervio periférico. Madrid: Graphia Eds. 1971. [Consulta: 2010-07-03].

6. KARLSSONA S. y Cols. Mean frequency and signal amplitude of the surface EMG of the quadriceps muscles increase with increasing torque - a study using the continuous wavelet transform, Journal of electromyography and Kinesiology. Volume 11, Issue 2, April 2001. [Consulta: 2010-08-12]. p. 131-140.

7. DANE. Resultados preliminares de la implementación del registro para la localización y caracterización de las personas con discapacidad Bogotá. 2007. [Documento electrónico] URL: http://www.dane.gov. co/files/investigaciones/discapacidad/Bogota\%20abril\%202005.pdf [Consultado el 2010-02-20].

8. ROMERO, A. JUGO, D. y Cols. Diseño e implementación de un instrumento virtual para la adquisición y procesamiento de señales fisiológicas. [Documento electrónico] URL: http://ciecfie.epn.edu.ec/jiee/ historial/XIXJIEE/5Perfiles_CIERHI.pdf [Consultado el 2010-03-20].

9. BETANCOURT, G., GIRALDO, E. y Cols. Reconocimiento de patrones de movimiento a partir de señales electromiográficas. [Documento electrónico]. URL: http://redalyc.uaemex.mx/src/inicio/ArtPdfRed. jsp?iCve=84911640010 [Consulta: $2010-03-07]$.
10. AGUILAR ZAMBRANO, J. Detección de señales mioeléctricas y su análisis con Redes Neuronales. [Documento electrónico]. URL: http://drupal.puj.edu.co/files/OI175_Jaime\%20Aguilar.pdf [Consulta: 2010-02-10].

11. RECUERO A. La Domótica como medio para la vida independiente de discapacitados y personas de la tercera edad, Informes de la Construcción, Vol. 50, No 459.1999.

12. Fatronik. [Documento electrónico]. URL: www.fatronik.com/noticia. php?id=97 [Consulta: 2010-03-07].

13. Iriscom. [Documento electrónico]. URL: www.iriscom.org/Noticias/ iriscom.html [Consulta: 2010-03-07].

14. Proinssa. [Documento electrónico]. URL: http://www.proinssa.com/ frames_seccion/oo_index_seccion.html?num $=05 \&$ nom $=$ domotica [Consulta: 2010-02-10].

15. González, E. y Cols. Estudio de factibilidad de un Mouse controlado por señales Electrooculográficas. UNMdP, Facultad de Ingeniería, UAH, Escuela Politécnica. XV Congreso Argentino de Bioingeniería. [Documento electrónico]. URL: http://www.sabi.org.ar/anales/ cd_2005/pdf/054PS.pdf [Consulta: 2010-08-12].

16. Brain Computer Interface. [Documento electrónico]. URL: http:// www.tech-faq.com/brain-computer-interface.html [Consulta: 201008-12].

17. ABBRUZZESE, G. et al. Response of arm flexor muscles to magnetic and electrical brain stimulation during shortening and lengthening tasks in man. Madrid, España. 2007. [Documento electrónico]. URL: http://www.ncbi.nlm.nih.gov/pmc/articles/PMC1155948/ [Consulta: 2010-03-08].

18. SANZ JUEZ C. y Cols. Domótica accesible. Dossier. 2006. [Documento electrónico]. URL: http://www.anobium.es/rdr. php?tema $=8 \&$ cat $=0 \&$ id $=139 \&$ id_alone $=139$ [Consulta: 2010-07-12] . p. 18-20.

19. DOMíngUEZ H. y Cols. Domótica: Un enfoque sociotécnico. CeDint, Centro de Domótica Integral. Junio 2006. ISBN: 847402-335-1 ed. Fundación Rogelio Segovia para el Desarrollo de las Telecomunicaciones Ciudad Universitaria, Madrid. [Consulta: 201006-10].

20. ELOY, S. y Cols. Integration of Information, Communication and Automation technologies in Housing Rehabilitation. XXXVII IAHS 
World Congress on Housing Oct 26 - 29, 2010. Santander, España. [Consulta: 2010-06-10].

21. STEGEMAN D. y Cols. Standards for surface electromyography: the European project Surface EMG for non-invasive assessment of muscles (SENIAM). Institute of Neurology, Department of Clinical Neurophysiology. 1999. [Consulta: 2010-06-10].

22. RUIZ, A. F., BRUNETTI, F y Cols. Adquisición y procesado de información EMG en el modelado de sistemas biológicos. Madrid, España. 2007. [Documento electrónico]. URL: http://www. physiologicalmeasurement/Adquisicion [Consulta: 2010-03-08].

23. TORRES, L. Tele-operación e Interfaces Hombre-Máquina TIHM. [Documento electrónico]. URL: http://bibliotecnica.upc.es/e-portals/ tid/arxius/articles/article_4.pdf [Consulta: 2010-02-10].

24. MUÑOZ RENGIFO, J. et. al. Adquisición de señales EMG, detección de movimientos de extensión y flexión. Universidad del Cauca, Popayán. 2008.

25. FELZER, T., FREISLEBEN, B. An Input Method for Human-Computer Interaction based on Muscle Control. Alemania. 2002.

26. GASCA D., ROJAS L. Extracción de Características Descriptoras de Movimientos de la Mano a Partir de Señales Electromiográficas (EMG) aplicando técnicas Wavelets. Universidad del Cauca, 2007.

27. ENGLEHART K., HUDGINS B., y Cols. Continuous multifunction myoelectric control using pattern recognition. Technology and disability. 2003. [Consulta: 2010-06-10]. 15:95-103.

28. ENGLEHART K., HUDGINS B., y Cols. Classification of the Myoelectric Signal using Time-Frequency Based Representations. Medical Engineering and Physics. Special Issue: Intelligent Data Analysis in Electromyography and Electroneurography. Vol. 21. 1999. [Consulta: 2010-06-10]. p. 431-438.

29. FERGUSON S., DUNLOP R. Grasp Recognition from Myoelectric Signals. Australasian Conference on Robotics and Automation, 2002.
30. GRAUPE D. EMG pattern analysis for patient-responsive control of FES in paraplegics for walker-supported walking. IEEE Transactions on Biomedical Engineering, Vol. 36. 1989. [Consulta: 2010-06-10].

31. VANEGAS, Diego. FONSECA, Santiago. Diseño de una interfaz hombre-máquina utilizando músculo masetero. Proyecto de grado. Universidad Central. Bogotá, 2007.

32. ESCUDERO J., PARADA M., y Cols. Amplificadores de instrumentación. Universidad de Sevilla. 2006. [Consulta: 2010-02-10]. p.1-10.

33. FARRY K., WALKER I., y Cols. Myoelectric Teleoperation of a Complex Robotic Hand. IEEE Transaction On Robotic and Automation, Vol. 12, 1996. [Consulta: 2010-06-10].

34. KUMAR S.y Cols. Effect of Noise on Facial EMG. Proceedings of the ACOUSTICS, 2004. [Consulta: 2010-02-10].

35. MERLETTI R., PARKER P. Electromyography: Physiology, engineering, and noninvasive applications. Vol. 11. IEEE Press series in Biomedical Engineering. John Wiley \& Sons, Inc., Hoboken, $3^{\circ}$ edition, New Jersey. 2004 .

36. ARENY R. Adquisición y distribución de señales. Marcombo, $3^{\circ}$ edition, México. 1993

37. Analog Devices. Instrumentation Amplifier AD620, 1999. [Documento electrónico]. URL:http://www.analog.com [Consulta: 2010-04-10].

38. ÁLVAREZ C. Seguridad eléctrica. Instrumentación biomédica. Universidad de Alcalá. 2004.

39. Biopac Systems, Inc. [Documento electrónico]. URL: www.biopac. com [Consulta: 2010-06-10].

40. HERRERA A., JARAMILLO R., y Cols. sEMG para control de prótesis Mioeléctricas. Escuela de Ingeniería de Antioquía (EIA) e Instituto de Ciencias de la Salud (CES). 2006.

41. FRERIKS B., H. HERMENS. European Recommendations for Surface ElectroMyo-Graphy. Roessingh Research and Development. 2000. [Consulta: 2010-06-10]

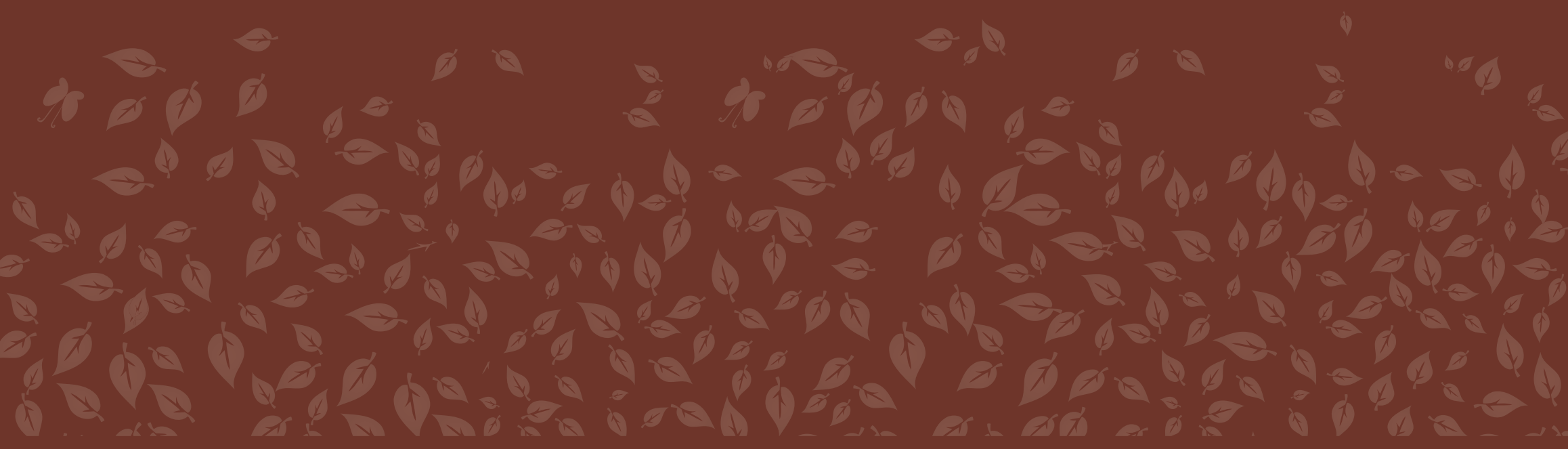

REV. COL. REH || Bogotá, Colombia || Volumen 10 || Página 86 - 93 || Noviembre 2011 || ISSN 1692 - 1879 